\title{
An Attitude Control System for the Deployment and Stabilisation of a Tethered Dual CubeSat Mission
}

\author{
Mike-Alec Kearney* Willem H. Steyn ** \\ * Electronic Systems Laboratory, University of Stellenbosch, \\ Stellenbosch, South Africa (e-mail: mkearney@sun.ac.za) \\ ** Electrical and Electronic Engineering Department, University of \\ Stellenbosch
}

\begin{abstract}
The use of electrodynamic tethers on board satellites is a promising scientific prospect. While the theory describing such systems has been studied by numerous scientists and mathematicians, practical testing of these systems has been relatively unsuccessful and test data concerning the topic is limited. Low-cost CubeSats offer a cheap platform from which practical tests can be done which would contribute to advancements in the field. The design of an attitude determination and control system for such a CubeSat tether mission is discussed in this paper. This includes the design of a sensor capable of measuring the orientation of the tethered CubeSat pair using a CMOS imager. Further, the study includes the design of an active deployment system, mathematical modelling of the system, formulation of control-algorithms for controlling the tether orientation, and full orbital simulations of the system.
\end{abstract}

\section{INTRODUCTION}

The concept of tethers in space has been around since early 1960 when Yuri Artsutanov, a Russian engineer, suggested a space elevator system where a satellite would lower a tether from geo-stationary orbit onto the ground. Thereafter, uses for tethers evolved and recently the concept of the Electrodynamic Tether (EDT) emerged.

An EDT is a conductive tether which interacts with the geomagnetic field. An electrical current flowing through the tether interacts with the geomagnetic field to generate a force which can be used to propel or de-orbit a tethered satellite system.

The electrodynamic principles have been successfully demonstrated on-board shuttle missions STS-46 and STS75 and later on board a large satellite mission named PMG. Multiple other unsuccessful missions have been attempted, but only these were considered to have provided reliable scientific results.

The next step in the process of developing the concept of EDTs is verification of the theoretical models used for describing the dynamics of the orbiting tether system, and verifying the electron collection theory which is currently used to model the electrical current in the tether. To do this, practical measurement data is required, which in turn requires more tether missions to be launched. The low success rate of tether missions, combined with the high cost of launching a satellite, makes the proposition of launching tethered satellites less attractive. It is necessary to prove that tethered satellites hold enough potential advantages and valuable uses to justify launching these missions.

By using low-cost CubeSats, however, many complex or high-risk satellite missions can be justified, considering the low cost of building and launching these Nano-satellites. The main challenge of integrating a tether system into a CubeSat is miniaturizing all components to fit within the size and weight limits of a nano-satellite.

The focus of this study is specifically on the attitude control of a tethered dual CubeSat system in Low Earth Orbit (LEO). A bare tether with an anodic contactor and an orbital altitude of $350 \mathrm{~km}$ was chosen for its efficiency (Hastings [1992]). A sensor was developed which can measure the angle at which the tether is orientated relative to some fixed orbital reference. This measurement can then be used in the control of the tether orientation. In this study a CMOS imaging sensor is proposed which measures the angle the tether makes with the Nadir vector. Oscillations in this angle are then damped by using the electrodynamic force/torque on the tether. A Brushless DC-motor (BLDCmotor) is used to control the deployment process. Prototypes of the sensors and components of the tether system were developed, its performance tested and the results of these tests used in simulation of the entire tether system in orbit. The system dynamics were simulated using adapted mathematical equations from Williams [2010], and the electrodynamics were simulated using analytical current collection equations derived by Claudio et al. [2010].

\section{SYSTEM DYNAMICS}

\subsection{Equations of motion}

To analyze the dynamics of the tethered system a mathematical model of the system is required. This model should be simple enough to be used in the derivation of a control law and an estimator without the system becoming of an impractically high order and complexity. The equations should, however, simultaneously model the system to sufficient accuracy. 
The massive slack-spring model was chosen. The main constraint of using this model is the fact that the forces associated with the lateral bending and oscillations of the tether is not considered. This model is specifically accurate for very thin and lightweight tethers. It is therefore well suited for CubeSat missions where thin tethers are used because of the volume and mass constraints of such Nanosatellites.

The state of the tether system is described by the tether length $\mathrm{L}$, the in-plane angle $\theta$ and the out-of-plane angle $\phi$ as shown in Fig. 1. The vector definitions made here are:

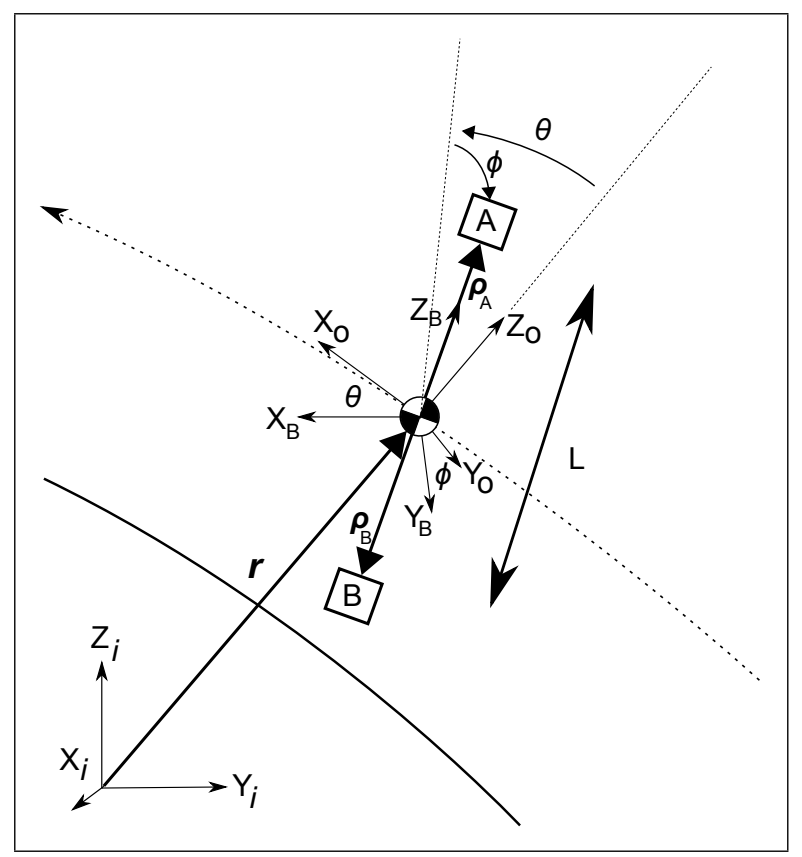

Fig. 1. Vector definitions

- $\mathbf{X}_{i}, \mathbf{Y}_{i}, \mathbf{Z}_{i}$ form the orthogonal inertial vector set

- $\mathbf{X}_{o}, \mathbf{Y}_{o}, \mathbf{Z}_{o}$ form the orthogonal orbital vector set

- $\mathbf{X}_{b}, \mathbf{Y}_{b}, \mathbf{Z}_{b}$ form the orthogonal body vector set

- $\boldsymbol{\rho}_{\boldsymbol{A}}$ is the vector from the CoM (Centre of Mass of total tethered orbiting system) to satellite $\mathrm{A}$ and is also written as $\boldsymbol{\rho}_{\boldsymbol{A}}=-\rho_{A} \hat{\boldsymbol{e}}_{\boldsymbol{\rho}}$ where $\hat{\boldsymbol{e}}_{\boldsymbol{\rho}}$ is the unit vector in the direction from satellite $B$ towards satellite A

- $\boldsymbol{\rho}_{\boldsymbol{B}}$ is the vector from the CoM to satellite B and is also written as $\boldsymbol{\rho}_{\boldsymbol{B}}=-\rho_{B} \hat{\boldsymbol{e}}_{\boldsymbol{\rho}}$

- $\boldsymbol{r}$ is the vector from the centre of the earth to the $\mathrm{CoM}$

The Lagrangian in vector form is written as (Williams [2010]):

$$
\begin{aligned}
\mathcal{L}= & \frac{1}{2} M \dot{\boldsymbol{r}} \cdot \dot{\boldsymbol{r}}+\frac{1}{2} \mu_{e}\left(\dot{L}^{2}+L^{2}\left(\boldsymbol{\Omega} \times \hat{\boldsymbol{e}}_{\boldsymbol{\rho}}\right) \cdot\left(\boldsymbol{\Omega} \times \hat{\boldsymbol{e}}_{\boldsymbol{\rho}}\right)\right) \\
& +\frac{\mu_{g} M}{R}+\frac{\mu_{g} L^{2}}{2 R^{3}} \mu_{e}\left(3\left(\hat{\boldsymbol{\rho}} \cdot \hat{\boldsymbol{e}}_{\boldsymbol{r}}\right)^{2}-1\right)
\end{aligned}
$$

The following unitary transform direct cosine matrix is used to convert vectors in the orbit-frame to the bodyframe.

$$
\mathbf{D C M}=\left[\begin{array}{ccc}
\cos \theta & 0 & -\sin \theta \\
\sin \phi \sin \theta & \cos \phi & \sin \phi \cos \theta \\
\cos \phi \sin \theta & -\sin \phi & \cos \phi \cos \theta
\end{array}\right]
$$

Using the Euler-Lagrange equation on Eq 1 and applying Eq 2 on the result, we arrive at the libration equations for the 3 state variables.

$$
\begin{aligned}
Q_{\theta}= & \mu_{e} L^{2}\left(\cos ^{2} \phi \ddot{\theta}+2\left(\frac{\dot{L}}{L} \cos ^{2} \phi-\dot{\phi} \cos \phi \sin \phi\right) \cdot(\dot{\theta}+\dot{v})+\right. \\
& \left.\frac{3 \mu_{g}}{r^{3}} \cos ^{2} \phi \cos \theta \sin \theta\right) \\
Q_{\phi}= & \mu_{e} L^{2}\left(\ddot{\phi}+2 \frac{\dot{L}}{L} \dot{\phi}+\cos \phi \sin \phi\left((\dot{\theta}+\dot{v})^{2}+\frac{3 \mu_{g}}{r^{3}} \cos ^{2} \theta\right)\right) \\
Q_{L}= & \mu_{e} L\left(\frac{\ddot{L}}{L}-\dot{\phi}^{2}-\cos ^{2} \phi(\dot{\theta}+\dot{v})^{2}-\frac{\mu_{g}}{r^{3}}\left(3 \cos ^{2} \phi \cos ^{2} \theta-1\right)\right)
\end{aligned}
$$

Where $Q_{\theta}, Q_{\phi}$ and $Q_{L}$ are the non-conservative forces acting on the system. The $\phi=\theta=0^{\circ}$-attitude forms a stable equilibrium and is thus chosen as the nominal orientation of the tether (Palmer [2011], Larsen [2011]). The dominant force providing the stability of the system at this orientation is the gravity-gradient force. Other forces are, however, also present and are discussed next.

\subsection{Non-Conservative forces}

The non-conservative forces are the external forces acting on the system, which add or remove energy from it. These include aerodynamic forces, electrodynamic forces, and elastic forces within the slack spring. Other disturbance forces such as solar pressure, variations in the earth's gravitational field, the gravitational effect of the moon and others, and the respective torques they cause on the system, are however insignificantly small in comparison with the three forces mentioned above.. Further, analysis revealed that even the aerodynamic force acting on the system has an insignificantly small effect on the orientation of the satellite, and is not further discussed.

Electrodynamic Force: Classically the current and bias profiles of EDTs are calculated using numerical methods. This is because of the complex nature of the electron collection process in a plasma. These numerical methods are computationally intensive and not well suited for Nanosatellites. Claudio et al. [2010] formulated an analytical solution to this problem that has a claimed accuracy of within $5 \%$ of numerical solutions. These analytical solutions are used to calculate the current profile along the tether and thus also the resulting electrodynamic forces acting on the system. These solutions assume operation under Orbit Motion Limited (OML) theory conditions. In the case of tethers in LEO, the tether will remain in the OML region if the tether thickness radius stays below 3 mm (Lorenzini et al. [2000]).

The performance of an EDT operating in OML is mainly limited by its electron collection and dispersion capabilities. This is governed by the contact impedance between the tether to its surrounding plasma. To lower this impedance and increase the efficiency of the system, a hollow cathode plasma contactor is traditionally used. The availability of miniaturised contactors was found to be a problem and forms a key obstacle in the development process of tethered CubeSats.

For a tether system in a $350 \mathrm{~km}$ equatorial orbit the motional electric field across the tether was calculated as $0.3304 \mathrm{~V} / \mathrm{m}$. An average electron plasma density of $N_{e}=0.2 \times 10^{-12} \mathrm{~m}^{-3}$ and a $500 \mathrm{~m} \mathrm{x} 0.2 \mathrm{~mm}$ round copper 
tether was assumed. Using the equations from Claudio et al. [2010], the average current through the copper tether was found to be $15 \mathrm{~mA}$ when using a realistically small cathodic contactor which should be suitable for CubeSat missions. When the system has no contactor device, it is referred to as a floating EDT. In this case, currents are much smaller and the electrodynamic forces are weaker. For control purposes these two modes of operation, with and without the contactor, are used as the two levels of actuation.

Assuming equal satellite weights, the system has a COM located in the middle of the tether. The profile of the current flow through the tether is of such a nature that larger currents flow at the one end of the tether than on the other. The Lorentz force resulting from this current flowing in the vicinity of the geomagnetic field is thus also larger on the one end of the tether and causes an effective torque around the COM. The torque resulting from operating the system with and without the plasma contactor were found to be $\left|T_{o n}\right|=12.2 m N$ and $\left|T_{o f f}\right|=-9.6 \times 10^{-5} \mathrm{mN}$.

Tether Elasticity: The elasticity of the tether becomes a significant factor during the final part of the deployment process. During deployment the restrictive force on the tether is kept to a minimum by the deployment controller to ensure smooth deployment. When the full deployment distance is reached, the satellites have to be slowed to a halt and during this period the tension in the tether will cause it to stretch. The copper stretches elastically in relation with Young's modulus (Eq 6).

$$
F=\frac{E A_{0} \Delta L}{L_{0}}
$$

When the tether is stretched far enough, the yield point of copper is reached, and the copper will start deforming permanently. This occurs when the tension in the tether exceeds the yield strength of copper, which is $\Gamma_{\text {yield }}=$ $70 M P a$.

If, during deployment, the controller fails to control the tether deployment and the tether becomes fully unwound in an uncontrolled manner, the elastic forces in the tether would have to absorb all the kinetic energy that is still present in the motion of the satellites. Using the elastic limits of the copper tether and the momentum of the moving satellites, the maximum speed at which the uncontrolled tether can reach full deployment, without the tether breaking, is calculated(Eq 7).

$$
\dot{L}_{\max }=\sqrt{\frac{A_{0} L_{0} \Gamma_{\max }^{2}}{\mu_{e} E}}
$$

Using this speed as the maximum deployment speed for the tether, safe deployment can be guaranteed in the case where the tether deployment is accidentally not halted before it is fully unwound.

\section{SENSOR DESIGN AND TESTING}

\subsection{Tether Angle Sensor Design}

To be able to study and control the dynamics of the system a sensor is required to measure the angle the tether makes with some reference direction. This is achieved by placing a CMOS imager on the lower satellite and keeping its camera Zenith-pointing. A LED is placed on the higher satellite(Fig. 2). By measuring the position

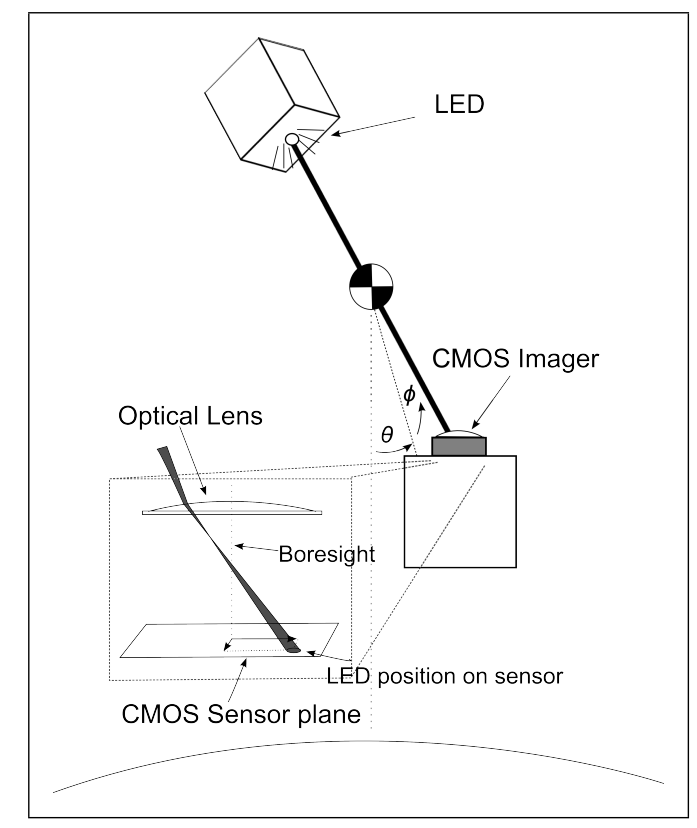

Fig. 2. Tether angle sensor conceptual design

of the LED in the image taken by the lower satellite, and assuming a straight tether, the tether in and outof-plane angle can be measured. It is important to note that if the satellite containing the CMOS sensor is not perfectly Zenith pointing, the tether angle sensor's output will also not be perfectly relative to the Zenith vector. This is however compensated for by accurately measuring the orientation of the CMOS sensor carrying satellite and using this in calculating the real angle the tether makes with the Zenith direction.

\subsection{Deployer Design}

The deployment mechanism requires an actuator for controlling the deployment speed, a sensor to measure the deployment distance and speed, and a release and guidance mechanism. A BLDC motor was chosen as and actuator for its high torque to size ratio. The deployment distance is measured using Hall sensor feedback on the BLDC motor. From these measurements the deployment speed is calculated. The guidance system consists of four linear bearings on parallel steel rods and a spring-loaded release mechanism(Fig. 3).

\subsection{Integration and Testing}

Electronic Design: $\quad$ To integrate the electronics needed to control the deployment speed, measure the tether angle, and to run estimation and control algorithms a single CubeSat sized PCB was designed containing all electronics(Fig. 4).

The electronics module was able to provide new angle measurements every 200ms and could run an Extended Kalman Filter (EKF) algorithm in $7 \mathrm{~ms}$.

The electronics and mechanical hardware built for the tether deployment prototype is shown in Fig. 5 


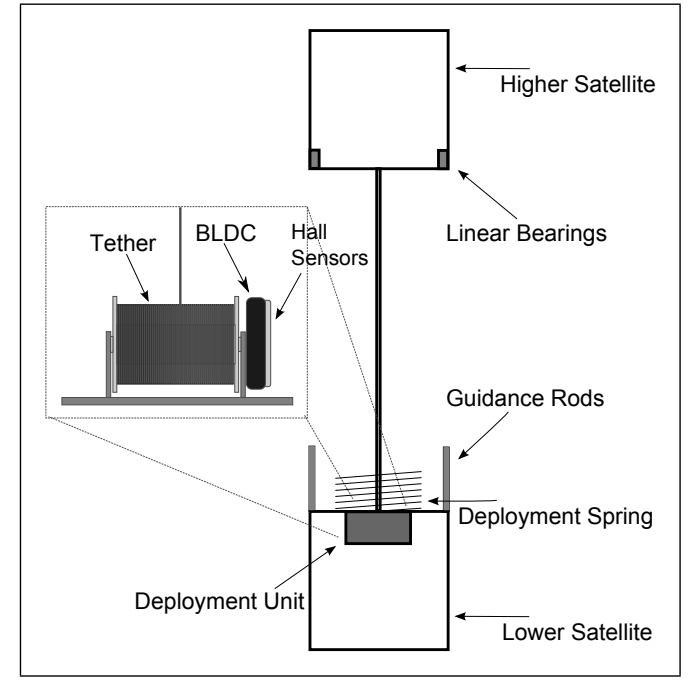

Fig. 3. Tether deployment controller conceptual design

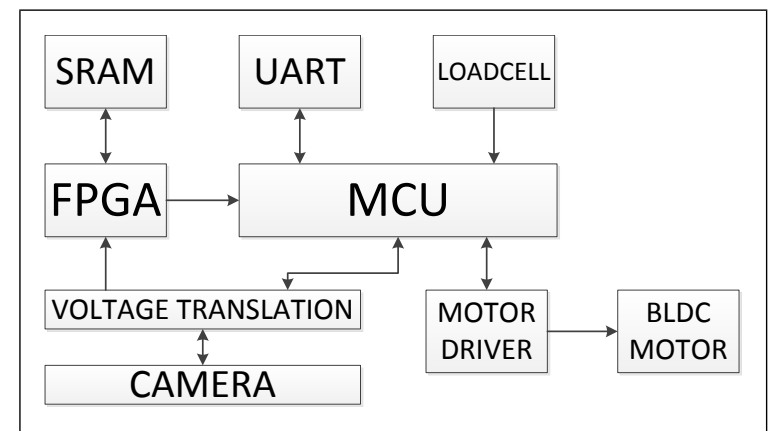

Fig. 4. Electronic interface summary

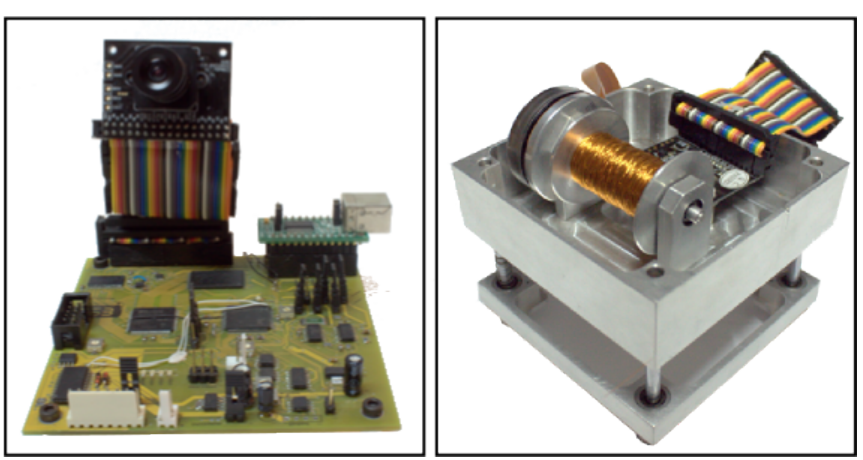

Fig. 5. CubeSat tether deployment hardware

\section{CONTROL}

\subsection{Extended Kalman Filter}

The system states have to be determined with the limited sensors available. To do this an EKF is used. The EKF uses the deployment distance and the tether angle as inputs to estimate the other states of the system. The system state vector is defined as:

$$
\mathbf{x}=\left[\begin{array}{llllll}
\theta & \phi & L & \dot{\theta} & \dot{\phi} & \dot{L}
\end{array}\right]^{T}
$$

Using Equations 3 to 5, the state equation is written as:

$$
\dot{\mathbf{x}}=\left[\begin{array}{c}
\dot{\theta} \\
\dot{\phi} \\
\dot{L} \\
-2\left(\dot{\theta}+\omega_{o}\right)\left(\frac{\dot{L}}{L}-\dot{\phi} \tan \phi\right)-3 \omega_{o}^{2} \cos \theta \sin \theta \\
-2 \frac{\dot{L}}{L} \dot{\phi}-\cos \phi \sin \phi\left(\left(\dot{\theta}+\omega_{o}\right)^{2}+3 \omega_{o}^{2} \cos ^{2} \theta\right) \\
L \dot{\phi}^{2}+L \cos ^{2} \phi\left(\dot{\theta}+\omega_{o}\right)^{2}+L \omega_{o}^{2}\left(3 \cos ^{2} \phi \cos ^{2} \theta-1\right)
\end{array}\right]+\mathbf{W}_{\mathbf{t}}
$$

where $\mathbf{W}_{\mathbf{t}}$ is zero-mean Gaussian process noise with covariance matrix of $\mathbf{Q}_{\mathbf{t}}$. The measurement equation can be written as

$$
\mathbf{y}=\mathbf{h}(\theta, \phi, L)+\mathbf{V}_{\mathbf{t}}=\left[\begin{array}{l}
\theta \\
\phi \\
L
\end{array}\right]+\mathbf{V}_{\mathbf{t}}
$$

where $\mathbf{V}_{\mathbf{t}}$ is zero mean Gaussian measurement noise with covariance matrix of $\mathbf{R}_{\mathbf{t}}$. Now the Jacobian of both the state matrix and the measurement matrix in Eq 9 and 10 can be calculated. The EFK algorithm can be executed using these matrices together with the noise covariance matrices, and the algorithm described in Haim [1999].

\subsection{Tether Angle Control}

The control of the tether angle is done by controlling the current through the tether and so controlling the electrodynamic force on the tether. To simplify the control design an on-off/bang-bang controller was used. This can be achieved by simply placing a power FET-switch just before the plasma contactor. When the switch is on and the plasma contactor is connected to the tether, the tether will have good contact with the surrounding plasma and the tether current will be a maximum. When the switch is off, the contact with the plasma is broken and the floating EDT will have a minimum current. The size of the current is proportional to the electrodynamic force/torque on the tether. Changing the contact with the plasma not only influences the size of the current, but also the current profile. This influences the position along the tether where the average force is and thus also the torque on the system. The analytical solutions for the current profile described earlier is used to do the calculations and determine the size and direction of the torque on the system. The decision of whether the switch should be toggled is made on the basis of whether the electrodynamic torque which will result at the present tether orientation would increase or decrease the tether oscillations. This control strategy is summarised in the Table 1

Table 1. *Tether angle control strategy

\begin{tabular}{|c||c|c|c|c|}
\hline & $\begin{array}{c}T_{\phi}>0 ; \\
T_{\theta}>0\end{array}$ & $\begin{array}{c}T_{\phi}>0 ; \\
T_{\theta}<0\end{array}$ & $\begin{array}{c}T_{\phi}<0 ; \\
T_{\theta}>0\end{array}$ & $\begin{array}{c}T_{\phi}<0 ; \\
T_{\theta}<0\end{array}$ \\
\hline \hline$\dot{\phi}>0 ; \dot{\theta}>0$ & & $\theta$ & $\phi$ & $\phi$ and $\theta$ \\
\hline$\dot{\phi}>0 ; \dot{\theta}>0$ & $\theta$ & & $\phi$ and $\theta$ & $\phi$ \\
\hline$\dot{\phi}>0 ; \dot{\theta}>0$ & $\phi$ & $\phi$ and $\theta$ & & $\theta$ \\
\hline$\dot{\phi}>0 ; \dot{\theta}>0$ & $\phi$ and $\theta$ & $\phi$ & $\theta$ & \\
\hline
\end{tabular}

*The table indicates which angles are damped for combinations of torque and angular rate. 
When the torque generated by the tether current decreases both the in and out-of-plane angle oscillations, the current is switched on. When it decreases only one of the two angles, a weighting system is used to prioritise the importance of decreasing a certain angle to determine if the tether current should be switched on or not.

\subsection{Deployment Control}

The strategy for the deployment control is based on a non-linear inversion control method combined with a PID regulator. Eq 5 can be rewritten as a function to calculate the acceleration in the tether direction.

$$
\ddot{L}=f_{L}(\mathbf{x})
$$

The control law implemented is then given by

$U_{L}(t)=K_{n} \cdot f_{L}^{-1}(\mathbf{x})+K_{p} \cdot e_{L}(t)+K_{i} \cdot \int_{0}^{\tau} e_{L}(\tau) \cdot d \tau+K_{d} \cdot \dot{e}_{L}(t)$

By using the inversion method, the highly non-linear system is linearised. The PID regulator then provides a computationally simple controller for controlling the deployment to a reference speed.

\subsection{Body rotations}

The effect of the taught tether on the body rotations of the individual CubeSats, and visa versa, is not considered in the model of the tether dynamics. The reason for this is that the size and mass as well as the energy in the rotations of the satellites are insignificantly small in comparison with the energy in their movement relative to each other. Another reason for not including these degrees of freedom, is that it causes the complexity of the libration equations describing the motion of the system to become extremely high. The argument is made that these effects will be small enough for the satellite to counter using it's own ADCS. The tether and satellite's dynamics are thus decoupled. The tether pulling on the satellite will however cause disturbance torques on the satellite bodies. The ADCS capabilities required by the individual satellites are set in line with these disturbance torques, so that the satellite will have sufficient control authority to mitigate these effects. The disturbance torque caused by the tether is derived as

$$
\mathbf{T}_{\text {tether }}=\frac{1}{2} d \cdot F_{\text {tether }} \cdot\left(\hat{\mathbf{e}}_{\text {body }} \times \hat{\mathbf{e}}_{\text {tether }}\right)
$$

Where $\mathrm{d}$ is the distance between the connection point of the tether and the COM of the satellite, and $F_{\text {tether }}$ is the tension in the tether. A miniature 3 axis reaction wheel ADCS suite in combination with magnetic control was found to be sufficient to stabilise the satellites under these disturbance conditions. Quaternion feedback control laws discussed in Steyn [1995] were used to keep the satellite earth-pointing in spite of the disturbances from the tether.

\section{SIMULATION AND RESULTS}

The deployment of the satellite was simulated in MATLAB Simulink. The simulation environment was adapted from work done by Steyn [1995]. In the following simulation the satellites were launched in opposite directions with one going towards Zenith and one towards Nadir. They were launched at speeds varying between $0.2 \mathrm{~m} / \mathrm{s}$ and $0.8 \mathrm{~m} / \mathrm{s}$ and the deployment controller was set to maintain this speed. The reference deployment distance was set to $480 \mathrm{~m}$. From these simulations it became clear that the system will always have some oscillations in the In-Plane angle after deployment. The reason is attributed to the conservation of angular momentum of the system in orbit. To maintain the orbital angular momentum the system has before deployment, the satellite that is losing altitude needs an increase in angular rate and the satellite gaining altitude needs a decrease in angular rate. This results in the lower satellite gaining a leading in-plane angle during deployment. After the deployment is stopped, gravity gradient pulls the tether back to the Nadir orientation resulting in an oscillation. Fig. 6 illustrates how the

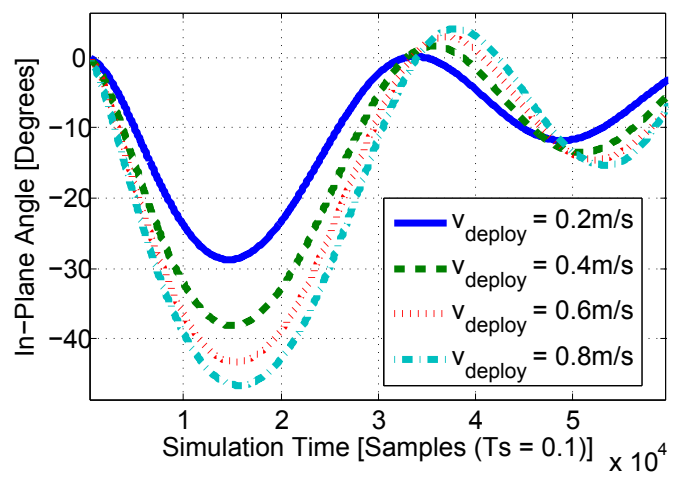

Fig. 6. Residual oscillation after deployment

deployment speed determines the size of this oscillation. To control the deployment speed, the controller discussed earlier and traditional PID controllers were implemented and compared. The non-linear dynamics of the system caused the pure PI controllers to oscillate, while the PI controller combined with the EKF and non-linear inversion control followed a speed reference with very small error.

The tether angle oscillations resulting after deployment are then damped in the simulation using the on/off-controller described earlier. Fig. 7 and Fig. 8 respectively show how
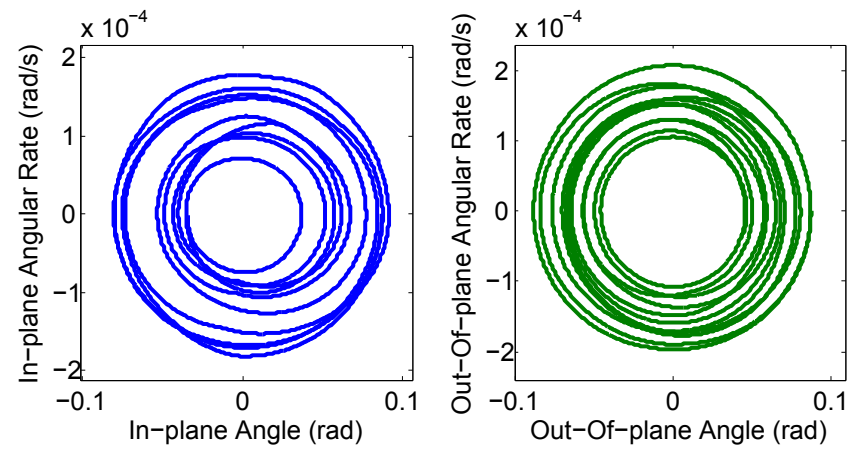

Fig. 7. Tether angles and rates(Equal control weights)

the tether angle controller can either drive both tether angles to the equilibrium position, or more aggressively control one of the tether angles(the in-plane angle in this case).

The magnitude of the electromagnetic force is low in comparison with the amount of energy present in these 

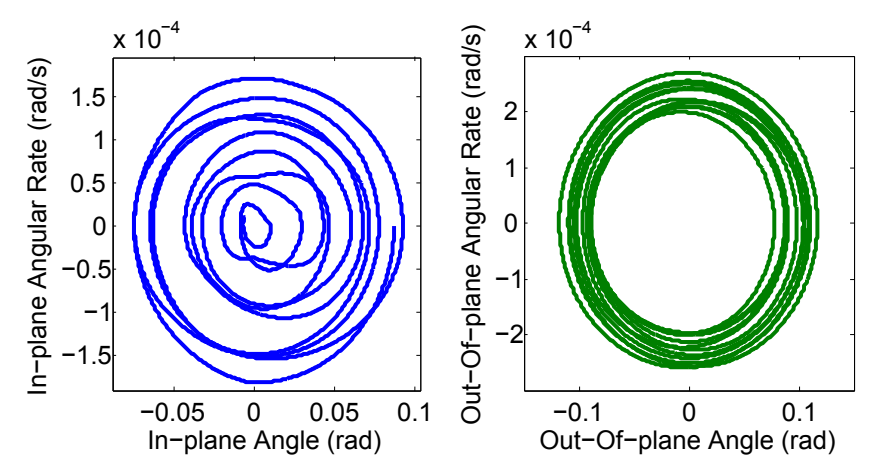

Fig. 8. Tether angles and rates (Larger in-plane angle control weight)

oscillations, and the oscillations can thus be damped only over a large period of time.

The effect the tether tension has on the orientation of the main satellite increases with these oscillations in the in-plane angle. The ADCS system's ability to compensate for the disturbance forces are simulated to ensure that the miniaturised reaction wheels have the torque and momentum capabilities to successfully keep the main satellite Nadir-pointing. From Fig. 9 it can be seen that the

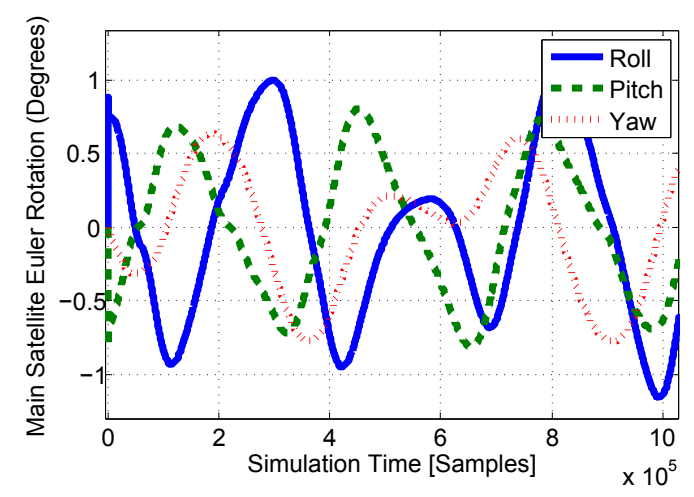

Fig. 9. Satellite body angles

miniaturised ADCS system is capable of keeping the main satellite earth pointing to within $1^{\circ}$ during deployment at $0.6 \mathrm{~m} / \mathrm{s}$.

\section{CONCLUSION}

The system dynamics where modelled and from this model an EKF algorithm was designed. This EKF is used to estimate the state variables used in the control algorithms for both the tether deployment and the tether angle. To execute the EKF and estimate the system states, a miniature sensor, capable of measuring the tether orientation angle, was needed. A CMOS sensor was designed and built and provided an accuracy better than one degree. For deployment a BLDC motor with Hall sensors is suggested as control hardware. In space, the forces the motor would have to exert on the tether would be small and a miniature BLDC motor can be used.

Using available analytical solutions for modelling of the electron collection process, the electrodynamic forces and torques on the tether could be calculated. The magnitude of the forces generated by the electrodynamic effect was found to be relatively small. These forces can thus not be used to do aggressive, accurate control of the tether orientation. Instead, it can be used to damp oscillations in the tether angle and so to control the tether to the Nadir equilibrium. The availability of miniaturised plasma contactors was found to be an obstacle in the process of developing a CubeSat tether system which will be able to generate significant currents.

The design was verified through simulation. It was found that a passive deployment strategy would not guarantee stable deployments and would not serve as reliable deployment mechanism. Stable deployment could be done using only PI control. The deployment process did however contain oscillations due to the non-linearity of the dynamic model. When using a full state EKF to estimate the system states, and inversion control methods to cancel the nonlinearity in the system, a stable, controllable deployment procedure was possible.

It was demonstrated through simulation that the effect the tether has on the orientation of the satellite can be mitigated using ADCS on each CubeSat, without having to model these effects. After simulation of the attitude of the individual CubeSats connected with the tether, it was found that the satellites could be kept Nadir pointing to within 2 degrees using standard CubeSat ADCS equipment.

During this study, the feasibility of implementing an EDT in a CubeSat was verified. Using the miniaturised ADCS components designed in the study, along with the control algorithms, stable deployment and control of the tether attitude could be done.

\section{REFERENCES}

D.E. Hastings, E. Ahedo, R.I. Samanta. Systems Analysis of Electrodynamic Tethers. Journal of spacecraft and rockets, 29 No 3: 1992

C. Bombardelli, J. Pelez, M. Sanjurjo. Asymptotic Solution for the Current Profile of Passive Bare Electrodynamic Tethers. Journal of Propulsion and Power, 26:1291-1304, 2010.

R.D. Lorenzini, E.C. Sanmart-egrave, J. Pel-uuml, J. Mart-egrave, M. Johnson, C.L. Vas, I.E. Estes. Bare Tethers for Electrodynamic Spacecraft Propulsion. Journal of Spacecraft and Rockets, $37 \mathrm{Vol} 2,2000$

H.E. Loubser, W.H. Steyn The development of Sun and Nadir sensors for a solar sail CubeSat. M.Sc Thesis, 2010.

W.H. Steyn. A Multi-mode Attitude Determiniation and Control System for Small Satellites Ph.D Thesis, 1995

P Williams. Optimal Control of Electrodynamic Tether Orbit Transfers Using Timescale Separation. Journal of Guidance, Control, and Dynamics, 12 No 4:979-998, 2010.

J. Manuel, N. Ayllon, J. Pelez, C. Bombardelli Performance Analysis Of Bare Electrodynamic Tethers As Microsat Deorbiting Systems. Advances in the astronautical sciences, 136 No 1: 2010

P. Palmer, M. Roberts, K.U. Kristiansen A unification of models of tethered satellites. Ph.D 2011

M.B. Larsen, M. Blanke. Journal of Guidance, Control, and Dynamics., 34 No 1:118-127, 2011

M. Baruh. Analytical Dynamics., 1st Edition, McGraw Hill, 1999 